\title{
Minimally Invasive Navigation for the Endovascular Treatment of Abdominal Aortic Aneurysm: Preclinical Validation of the Endovax System
}

\author{
Sonia Pujol ${ }^{1}$, Philippe Cinquin ${ }^{1}$, Matthieu Pecher ${ }^{2}$, \\ Ivan Bricault ${ }^{3}$, and David Voirin ${ }^{2}$ \\ 1 TIMC-GMCAO Laboratory, UMR CNRS 5525, Faculty of Medicine, \\ 38706 Grenoble, France \\ \{sonia.pujol\}@imag.fr \\ 2 Dept. of Vascular Surgery, Michallon Hospital, 38706 Grenoble, France \\ 3 Dept. of Radiology, Michallon Hospital, 38706 Grenoble, France
}

\begin{abstract}
Endovascular repairs are promising solutions for a minimally invasive treatment of Abdominal Aortic Aneurysms. This paper describes a new method for assisting surgeons in conducting endovascular procedures with a reduced X-rays exposure. An anatomical landmark-based registration between pre-operative $3 \mathrm{D}$ CT scan and transabdominal $2.5 \mathrm{D}$ Ultrasound images replaces the $\mathrm{CT}$ data in the intra-operative referential. A modified ICP algorithm computes the transformation between the internal surface of the aorta segmented from CT images and its position in ultrasound data. Magnetic tracking is performed to navigate a modified guidewire. The visualization module presents the location of the guidewire in multiplanar reformatted CT views and inside a 3D Model of the lumen. The relative position of the sensor is updated in real time from position data obtained from the magnetic tracking. We applied the method to an anatomical piece, and led an endovascular procedure with clinically satisfactory results.
\end{abstract}

\section{Introduction}

Abdominal Aortic Aneurysm (AAA) is a degenerative disease of the aortic wall, responsible for considerable morbidity and mortality. Endovascular surgery excludes the aneurysm by implanting a prosthesis inside the aorta from a transfemoral route of delivery [1. This provides the most appropriate minimally invasive solution for patients who have aneurysms at a significant risk of rupture, but who cannot bear traditional open surgical repair involving aorta clamping.

Endovascular Surgery requires the surgeon to operate based on a real-time imaging modality. Fluoroscopic guidance is the gold-standard to perform the endovascular repair: radio-opaque markers show the position of the endoprosthesis on the monitor of the image intensifier. This procedure has several pitfalls: the 
patient and the medical staff are exposed to X-rays, and the radiographic contrast medium is nephrotoxic. Moreover, angiography is a projective technique, which involves a mental reconstruction of the $3 \mathrm{D}$ structure of the vascular tree.

Our objective is to provide surgeons with a navigation system combining reduced X-rays exposure with tracking of the endovascular tools in a 3D model of the vessels.

Studies have reported the localization of endovascular devices in preoperative CT scans, by registration with intra-operative Digital Substraction Angiography images [2], but the exposure radiation was not reduced as the navigation was performed under fluoroscopic guidance. Less invasive imaging modalities may be of benefit to guide endovascular repair. Alternative localization procedures using Magnetic Resonance Imaging have been proposed [3] [4, but MRI systems are cumbersome and expensive, and surgical instruments have to be MRI compatible. Intravascular ultrasound provides high quality transversal images of the aneurysm, which may ensure precise delivery of the endovascular devices [5]. However the involved risks significantly limit its usage in AAA patients where the rupture can occur abruptly. Transabdominal Ultrasound imaging (US) is the standard method used to explore aneurysms, from diagnosis to post-operative supervision [6]. Investigators have demonstrated the feasibility of pre-operative CT and intra-operative US data fusion in minimally invasive abdominal surgery, based on anatomical and fiducial landmarks [7. However, transabdominal ultrasound cannot be used as a tracking tool to follow the progression of the endoprosthesis. Pre-operative Computed Tomography (CT) is routinely used for screening and planning the endovascular repair. The CT anatomical data are relevant until the delivery of the endoprosthesis. Our method proposes to virtually insert the location of the endoprosthesis inside the three-dimensional CT pre-operative images during the endovascular repair. The navigation system we have developed enables a real-time localization of the endoprosthesis thanks to registration between pre-operative CT scans, intra-operative 2.5D US data, and the magnetically tracked delivery device of an endoprosthesis. Experimental tests have been performed on an aortic phantom [8].

This article presents the first preclinical evaluation of the Endovax navigation system, and proposes a modified ICP algorithm for the registration between CT scans and US images.

\section{Material}

The preclinical experiment was set up with the following elements. A vascular guidewire (3F diameter, $260 \mathrm{~cm}$ long) equipped with a 5 DoF (Degrees of Freedom) magnetic sensor (0.8 mm diameter, $10 \mathrm{~mm}$ long), defining the $R_{\text {Sensor }}$ referential, was used to lead the endovascular procedure. The objective of the demonstration phase was to determine the position of this magnetic guidewire with respect to pre-operative CT scans. The modified guidewire was tracked by the Aurora ${ }^{T M}$ Magnetic Tracking System (Northern Digital Inc., Ontario, Canada). The position and orientation of the sensor in the $R_{\text {Aurora }}$ referential 
of the magnetic field generator were computed from the electric signal induced during its displacements. The experiment was led on an anatomical piece. A surgical removal of the organs in front of the aorta was performed on the trunk of a cadaver, and the anatomical piece was filled with ultrasound gel to enable further ultrasound examination. The arteries were injected with radiographic contrast medium dissolved in gelatine so as to enable opacification during preoperative Computer Tomography. An optical Rigid Body defining the $R_{O R}$ Operating Room referential was fixed on the operating table. Pre-Operative data consisted of CT scans of the anatomical piece acquired with a multi-slices CT Siemens Sensation 16 Scanner. The exam contained 400 slices of $1 \mathrm{~mm}$ thickness. A 3D isotropic volume was computed to define a $R_{C T}$ referential with a voxel size of $0.79 \mathrm{~mm}$. Intra-Operative data consisted of US scans acquired with a $3.5 \mathrm{MHz}$ Ultrasound sectorial probe equipped with an optical Rigid Body defining the $R_{U \text { SProbe }}$ referential, and tracked by a passive Polaris ${ }^{T M}$ Optical Localizer (Northern Digital Inc., Ontario, Canada). A 2.5D Ultrasound software application calculated for each image the transformation between the $R_{U \text { Simage }}$ reference system of the image and $R_{O R}($ Fig (1).

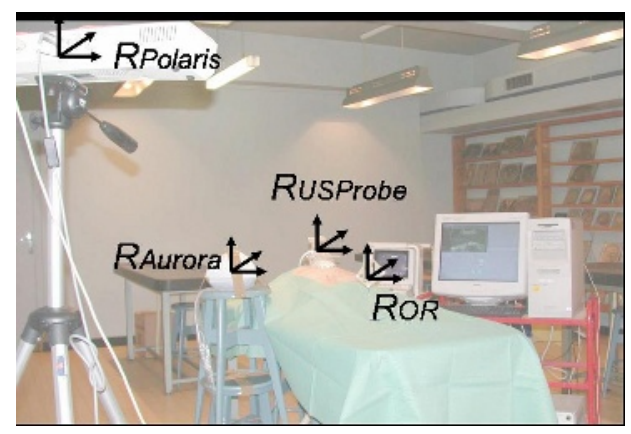

Fig. 1. Intra-operative elements of the navigation system.

\subsection{Methods}

The aim of the proposed method is the registration between the pre-operative CT scans localized in $R_{C T}$, and the intra-operative magnetic data defining the position of the endoprosthesis in $R_{O R}$. A paired-points registration is performed with the Arun method [9], and a modified Iterative Closest Point (ICP) algorithm is described.

Anatomical Landmarks Registration. Five strategic anatomical landmarks are localized in both pre-operative $R_{C T}$ and intra-operative $R_{O R}$ referentials: the aorto-iliac bifurcation, the ostia of the renal arteries, sub-mesenteric artery and celiac trunk. The anatomical landmarks are manually picked in 3D CT slices and 
US images. Thanks to the 2.5D Ultrasound system, the US landmarks localized in the referential $R_{U S_{\text {image }}}$ are replaced into the intra-operative absolute referential $R_{O R}$. A paired-points matching is performed using the Arun algorithm between the anatomical landmarks sets localized in $R_{C T}$ and in $R_{O R}$, which results in the initial attitude $T_{\left(R_{O R} \rightarrow R_{C T}\right)_{i}}$.

Modified ICP. Classical Iterative Closest Point (ICP) algorithm [10] computes the rigid transform that aligns a cloud of Scene points with a geometrical Model. A triangular set of points representing the internal surface of the aneurysm in $R_{C T}$ is computed automatically by a virtual exploratory navigation algorithm [1]. For each point of the CT surface of the aorta (Scene), we have to propose a corresponding point in the 2.5D US images (Model), but we would like to avoid the segmentation phase on ultrasound data.

The first step implies finding the intersection between US planes and CT volume. The CT mesh is replaced in the $R_{U \text { Simage }}$ referential of an ultrasound image $I_{U S_{i}}$ of the aneurysm by the transformation:

$$
T_{R_{C T} \rightarrow R_{U S i m a g e}}=T_{R_{U S \text { Probe }} \rightarrow R_{U S_{\text {image }}}} * T_{R_{O R} \rightarrow R_{U S_{P r o b e}}} * T_{\left(R_{C T} \rightarrow R_{O R}\right)_{i}}
$$

The equation of the ultrasound plane $\Pi_{i}$ in $R_{\text {Usimage }}$ is:

$$
\forall M(x, y, z) \in \Pi_{i}, z=0
$$

We select the set of the triangles

$$
\Delta_{i}=\left\{T_{i_{k}}\right\}_{k \in[0, \ldots, N]}
$$

whose segment endpoint z-coordinates change sign, and the intersection between the edges of $\Delta_{i}$ and $\Pi_{i}$ is computed. The resulting set of points

$$
\Omega_{i}=\left\{M_{i_{k}}\right\}_{k \in[0, \ldots, N]}
$$

represents the intersection of the triangular CT mesh with the US image plane (Fig. 2). The ultrasound edge between the thrombus and the circulating channel

$$
\Sigma_{i}=\left\{P_{i_{k}}\right\}_{k \in[0, \ldots, N]}
$$

is searched along the normals $\boldsymbol{n}_{\boldsymbol{i}_{\boldsymbol{k}}}$ to $\Delta_{i}$ from each point $M_{i_{k}}$ of $\Omega_{i}$.

The lumen creates a relatively homogenous low-intensity ultrasound signal, whereas the thrombus signal is inhomogeneous. From each point $M_{i_{k}}$ we compute the minima of a function

$$
f(u)=g\left(V_{f}, V_{b}\right)
$$

where $V_{f}$ and $V_{b}$ are the variances of two sets of pixels $\left\{u_{\text {front }}\right\}$ and $\left\{u_{\text {back }}\right\}$ located in the neighbourhood of the point of abscissa $u$ along $\boldsymbol{n}_{\boldsymbol{i}_{\boldsymbol{k}}}$. The point $P_{i_{k}}$ which corresponds to $M_{i_{k}}$ is the lowest minimum of $\mathrm{f}$ satisfying the criterium

$$
I\left(P_{i_{k}}\right) \leq \varepsilon_{L}
$$




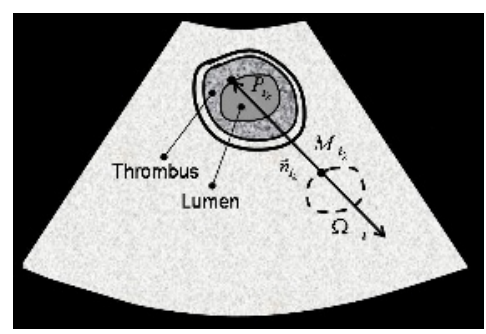

Fig. 2. The US corresponding point $P_{i_{k}}$ to the CT lumen point $M_{i_{k}}$ is searched along $\boldsymbol{n}_{\boldsymbol{i}_{\boldsymbol{k}}}$.

where $\mathrm{I}\left(P_{i_{k}}\right)$ is the ultrasound intensity of $P_{i_{k}}$, and $\varepsilon_{L}$ is a threshold for the lumen grey-level.

The Model is the edge $\Sigma_{i}=\left\{P_{i_{k}}\right\}_{k \in[0, \ldots, N]}$ replaced in $R_{O R}$ by the transformation:

$$
T_{R_{U S_{\text {image }}} \rightarrow R_{O R}}=T_{R_{U S_{\text {Probe }}} \rightarrow R_{O R}} * T_{R_{U S_{\text {image }}} \rightarrow R_{U S_{\text {Probe }}}}
$$

The Scene is the intersection $\Omega_{i}=\left\{M_{i_{k}}\right\}_{k \in[0, \ldots, N]}$ replaced in $R_{C T}$ thanks to the reverse transformation:

$$
T_{R_{U S_{\text {image }}} \rightarrow R_{C T}}=T_{R_{C T} \rightarrow R_{U S_{\text {image }}}}{ }^{-1}
$$

The ICP algorithm computes the transformation between the Scene and the Model resulting in the final registration $T_{R_{O R} \rightarrow R_{C T}}$.

Magnetic Tracking. The modified guidewire is tracked by the Aurora ${ }^{T M}$ system, and its position is calculated in real-time in $R_{C T}$ by the transformation:

$T_{R_{\text {Sensor }} \rightarrow R_{C T}}=T_{R_{O R} \rightarrow R_{C T}} * T_{R_{\text {Polaris }} \rightarrow R_{O R}} * T_{R_{\text {Aurora }} \rightarrow R_{\text {Polaris }}} * T_{R_{\text {Sensor }} \rightarrow R_{\text {Aurora }}}$

\section{Results}

\subsection{Preclinical Validation on the Anatomical Piece}

The evaluation consisted in two steps. First the clinician performed the endovascular procedure by introducing the magnetic guidewire in the right femoral artery, and used the Endovax system to navigate in the abdominal aorta under magnetic guidance. Each movement of the guidewire inside the vascular tree was reproduced in real-time in the $3 \mathrm{D}$ mesh of the lumen, and the system updated CT images by cutting in the 3D volume sagittal, transversal and longitudinal views centered on the magnetic sensor position. Secondly, once the clinician had 
reached strategic arteries ostia following the indications of the Endovax system, laparotomy was performed on the anatomical piece to compare the position indicated by the navigation system and the real position of the sensor in the aorta (Fig. 3) (Fig.4). The accuracy of the endovascular navigation system was quanti-

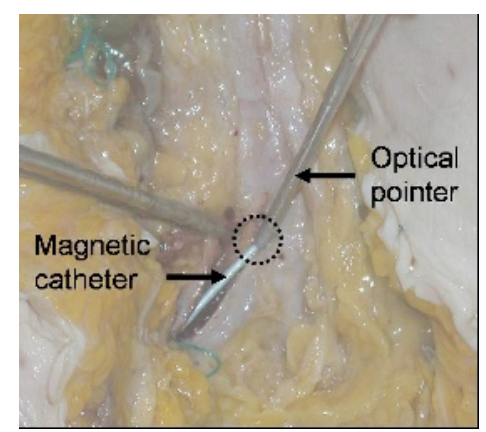

Fig. 3. Magnetic catheter and optical pointer in contact (dotted line) at the aorto-iliac bifurcation after laparotomy and vessel dissection.

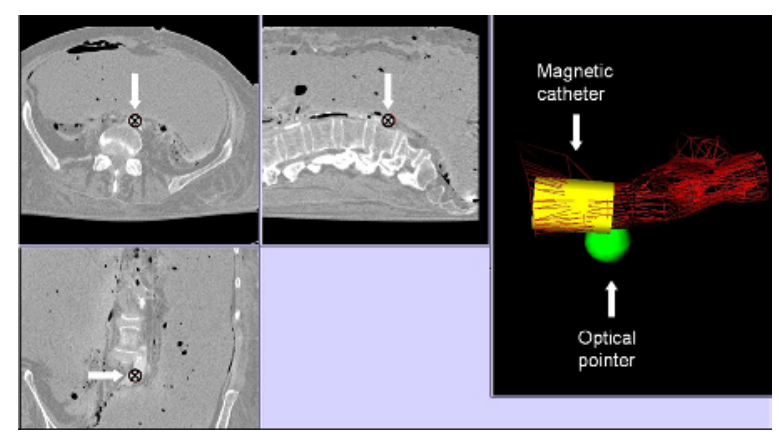

Fig. 4. ENDOVAX vizualisation module: transversal, sagittal and frontal slices of the pre-operative CT volume (left) and 3D model of the aorta (right). Arrows point towards the location of the magnetic catheter at the aorto-iliac bifurcation in CT slices and in 3D Model.

fied by the measurement of the distance between the magnetic catheter position and the anatomical objective. This distance was obtained by optical palpation. The measurements at the aorto-iliac bifurcation, left renal artery ostium, right renal artery ostium and inferior mesenteric artery ostium resulted in an average accuracy of $2.4 \mathrm{~mm}$ which is a clinically satisfactory result. 


\subsection{Preliminary Evaluation of the CT/US Registration on Patient Data}

The modified ICP algorithm has been tested on patient data. The CT mesh was replaced in $R_{O R}$ thanks to $T_{R_{O R} \rightarrow R_{C T}}^{-1}$ transformation, and the intersections of the CT lumen with US images were computed as described in Section 2.1 The registration accuracy was evaluated by computing the distance between the CT/US intersections and the lumen of the manually segmented aneurysm (Fig. 5). Preliminary results have demonstrated an average accuracy of $3.8 \mathrm{~mm}$.

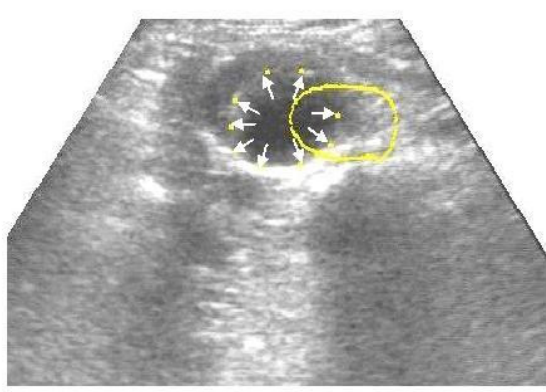

(a)

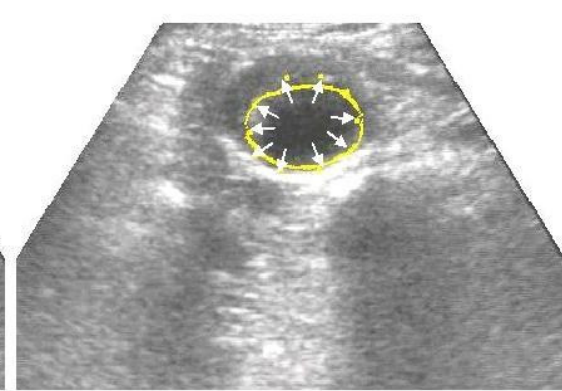

(b)

Fig. 5. Registration of the CT lumen position inside an ultrasound image of the aorta: (a) Arun initial estimate (b) Modified ICP. The results are compared with the manual segmentation done by a clinician (arrows).

\section{Discussion-Conclusion}

The feasibility of implanting endovascular devices by introducing them into the arterial tree relies on the ability to control the appropriate location of delivery. A magnetic guidance of endovascular devices in pre-operative CT images has been developed to assist surgeons in the treatment of Abdominal Aortic Aneurysms. Pre-operative data are replaced in the operating theater referential, using anatomical landmarks combined with 2.5D Ultrasound and optical localization. The experiment has demonstrated the preclinical validation of a new navigation procedure for assisting surgeons in positioning endovascular devices with reduced intra-operative use of fluoroscopy, and preliminary clinically satisfactory results. A modified ICP algorithm which takes advantages of the duality between segmentation and registration has been tested on patient data. We are presently quantifying the accuracy of the registration.

Future works will focus on the introduction of a model of ultrasound and the use of mutual information to improve the registration between CT scans and 
US images. Furthermore, first clinical tests will be performed to quantitatively compare traditional angiography versus CT magnetic navigation.

Acknowledgments. The authors thank M. Acosta and M. Haigron from the LTSI (Rennes, France), M. Thibaut from the UJF's Laboratory of Anatomy, PRAXIM Inc. (Grenoble, France), and Northern Digital Inc. (Ontario, Canada). This project was partly funded under the RNTS program of the French Ministry of Research (Project Surgetique II).

A colour-version of this article is located at the adress:

http://www-timc.imag.fr/Sonia.Pujol/

\section{References}

1. Faries, P.L.: Endovascular Grafts for the Treatment of Abdominal Aortic Aneurysm. Endovascular Grafting: Advanced Treatment for vascular disease, Endovascular Intervention: Today and Tomorrow, Vol.1.Futura Publishing., 73107,(2000).

2. Goksu, C., Haigron, P., Zhang, H., Soulas, T., Le Certen, G., Lucas, A.: 3D intraoperative localization for endovascular navigation guidance. Proceedings of Computer-Aided Medical Interventions: tools and app. Surgetica 2002.

3. Van Der Weide, R., Bakker, C., Viergever, M.: Localization of Intravascular Devices with Paramagnetic Markers in MR Images. IEEE Transactions on medical imaging, vol. 20, NO. 10, (2001).

4. Quick, H. H., Ladd M. E., Nans, D., Mikolajczyk, K. P., Debatin, J.F. Vascular Stents as RF antennas for intravascular MR guidance and imaging. Magn. Reson. Med., 1999 Oct, 42(4):738-45.

5. Garret HE, J.R., Abdullah, A.H., Hodgkiss, T.D., Burgar,S.R. Intravascular ultrasound aids in the performance of endovascular repair of abdominal aortic aneurysm. Journal of Vascular Surgery. 2003 Mar;37(3):615-8.

6. Sparks, A.R., Johnson, P.L., Meyer, M.C.: Imaging of Abdominal Aneurysms. Am. Fam. Physician. 2002 Apr 15;65(8):1565-70.

7. Sjolie, E., Kaspersen, J. H., Wesche, J., Lindseth, F., Hernes, T. A. N.: Minimal Invasive Abdominal Surgery based on Ultrasound Vision, possible? Proceedings of Computer Assisted Radiology and Surgery (CARS) 2001, 39-44.

8. Pujol, S., Pecher, M., Cinquin, P.: Magnetic guidance of endovascular devices in pre-operative CT images for the treatment of abdominal aortic aneurysm. Proceedings of Computer-Aided Medical Interventions : tools and app. Surgetica 2002.

9. Arun, K., Huang, T., Blostein, S.: LS fitting of two 3-D point sets. IEEE T-PAMI, 9(5), 1987.

10. Besl, P. J., McKay, N. D.:A method for registration of 3-D shapes. IEEE transactions on pattern analysis and machine intelligence, vol. 14, no 2, Feb. 1992.

11. Acosta, O., Moisan, C., Haigron, P., Lucas, A.: Evaluation of Virtual Exploratory Navigation for the Characterization of Stenosis in the Planning of Endovascular Interventions. SPIE Medical Imaging 2002, Physiology and function from multidimensional images, San Diego, USA, Feb. 2002, 4683, 42-53. 